Revue des patrimoines

40 | 2019

Lits historiques. Première anthologie des lits européens du XVe au XIXe siècle

\title{
Le vocabulaire et les typologies des lits à Paris d'après les inventaires après décès de la première moitié du XVII siècle
}

Bed Vocabularies and Typologies in Paris during the First Half of the

Seventeenth Century, According to Inventories after Death

\section{Nicolas Courtin}

\section{OpenEdition}

Journals

Édition électronique

URL : http://journals.openedition.org/insitu/23316

DOI : 10.4000/insitu.23316

ISSN : 1630-7305

Éditeur

Ministère de la Culture

\section{Référence électronique}

Nicolas Courtin, «Le vocabulaire et les typologies des lits à Paris d'après les inventaires après décès de la première moitié du xvı" siècle », In Situ [En ligne], 40 | 2019, mis en ligne le 15 septembre 2019, consulté le 04 octobre 2019. URL : http://journals.openedition.org/insitu/23316 ; DOI : 10.4000/insitu. 23316

Ce document a été généré automatiquement le 4 octobre 2019.

\section{(c) $(1) \odot$}

In Situ Revues des patrimoines est mis à disposition selon les termes de la licence Creative Commons Attribution - Pas d'Utilisation Commerciale - Pas de Modification 4.0 International. 


\title{
Le vocabulaire et les typologies des lits à Paris d'après les inventaires après décès de la première moitié du XVII ${ }^{\mathrm{e}}$ siècle
}

\author{
Bed Vocabularies and Typologies in Paris during the First Half of the \\ Seventeenth Century, According to Inventories after Death
}

Nicolas Courtin

$1 \mathrm{Au} \mathrm{XVII}{ }^{\mathrm{e}}$ siècle, le mot « lit » n'est pas le terme le plus courant pour désigner le meuble du sommeil.

2 Les dictionnaires de langue française établissent clairement que dans les vingt dernières années $d u \mathrm{XVII}^{\mathrm{e}}$ siècle, le mot « lit » s'est imposé pour désigner le meuble du sommeil dans son ensemble ${ }^{1}$, mais les notices font comprendre qu'il s'agit d'une évolution sémantique relativement récente. Auparavant, on distinguait en effet le « lit » et la « couche »; le premier désignait " particulièrement (...) le matelas et le lit de plume où l'on couche, (...) les draps et les couvertures qui y servent $»^{2}$, et le second la structure du meuble. Le vocabulaire reflète donc clairement la nature double de ce meuble où le bois et les textiles sont les deux éléments constitutifs et indispensables d'un même objet.

$3 \mathrm{Si}$, dans les années 1690, l'usage privilégiant le mot « lit » semble acquis, ce n'est pas le cas des actes notariés de la première moitié du siècle. L'examen des inventaires après décès parisiens de cette période ${ }^{3}$ fait apparaître un emploi dominant du mot " couche » pour désigner le meuble dans son ensemble. « Lit » apparaît, mais principalement pour désigner le coucher, c'est-à-dire dans son sens générique, partagé avec la géologie ou la maçonnerie, qui évoque les notions d'horizontalité et de superposition de couches.

4 Le lit $\mathrm{du} \mathrm{XVII}^{\mathrm{e}}$ siècle est donc un meuble en trois parties, le bois, le coucher et la garniture, qui relèvent chacune de natures et de métiers différents. Nous retiendrons cette division dans notre présentation du vocabulaire et des typologies. 


\section{Les bois et les différents types de lits}

Dans notre corpus, le squelette du meuble - ou «châlit» - est toujours en bois; d'ailleurs les notaires emploient bien, lorsqu'ils trouvent dans les garde-meubles des structures démontées, le terme "bois de lit». Ces travaux de menuiserie sont majoritairement en noyer, parfois en chêne et exceptionnellement en hêtre.

Le mot « couche » est donc le plus fréquemment employé. Il se décline en " couchette » pour signaler les meubles plus petits et plus spécifiquement, selon le dictionnaire de l'Académie (qui est le seul à proposer une définition pour ce terme), un meuble pour les enfants. «Lit » se décline quant à lui en différentes typologies : « lit de sangle » ou « lit brisé »(pliant) notamment, ce qui renvoie en revanche à d'autres manières de construire le meuble.

7 Couches, couchettes et lits trouvent tous des déclinaisons selon les dimensions estimées par les rédacteurs de l'inventaire. Ils peuvent être « grands » ou " petits » sans que l'on sache à quelles réalités cela correspond. Les couches peuvent également être "moyennes" ou "bâtardes", mais ces deux termes ne semblent pas synonymes, puisque l'on trouve la mention d'une « couche petite et bâtarde »...

Quelques rares inventaires donnent des mesures ${ }^{4}$; il s'agit en général des meubles les plus importants de la maison, rendant l'analyse de ces dimensions impossible à généraliser. Toutefois, il nous semble utile de rappeler les chiffres donnés. La longueur moyenne repérée est de 5,5 ou 6 pieds (soit entre 180 et $195 \mathrm{~cm}$ environ), pour des largeurs entre 4 et 6 pieds - mais majoritairement 5,5 pieds (soit environ $180 \mathrm{~cm}$ ). Aucune hauteur n'est mentionnée pour cette période. Il apparaît donc que les lits parisiens de la première moitié du siècle étaient d'un format presque carré.

Les descriptions trouvées dans les inventaires notariés sont généralement très stéréotypées. Après l'indication, même très vague, de la taille du meuble, on précise en général sa structure. On emploie alors exclusivement le mot de " pilier » pour désigner ce qu'on appelle aujourd'hui souvent les colonnes du lit, et on précise s'ils sont "hauts" ou "bas». Il faut comprendre cette distinction comme l'indication de la présence de supports pour un ciel de lit ou non : les hauts piliers soutiennent le " ciel ", alors que les bas piliers contiennent le coucher (fig. 1). Ce détail est précieux et permet de remarquer que tous les lits de cette époque ne sont pas des lits à baldaquin mais qu'il en existe d'autres types - plus simples ou surmontés d'un ciel indépendant de la couche ${ }^{5}$. Toutefois, l'absence de hauts piliers ne veut pas forcément dire que la couche n'est pas dotée d'une garniture permettant de la clore, comme il sera expliqué plus bas à propos des lits «à la romaine ». Notons enfin que la présence de montants hauts ou bas n'est pas liée à la taille du lit: les « couchettes » ou les «petites couches » peuvent être surmontées d'un ciel ou pas. En revanche, tous les grands lits et les couches dites «bâtardes » sont à « hauts piliers ». 
Figure 1

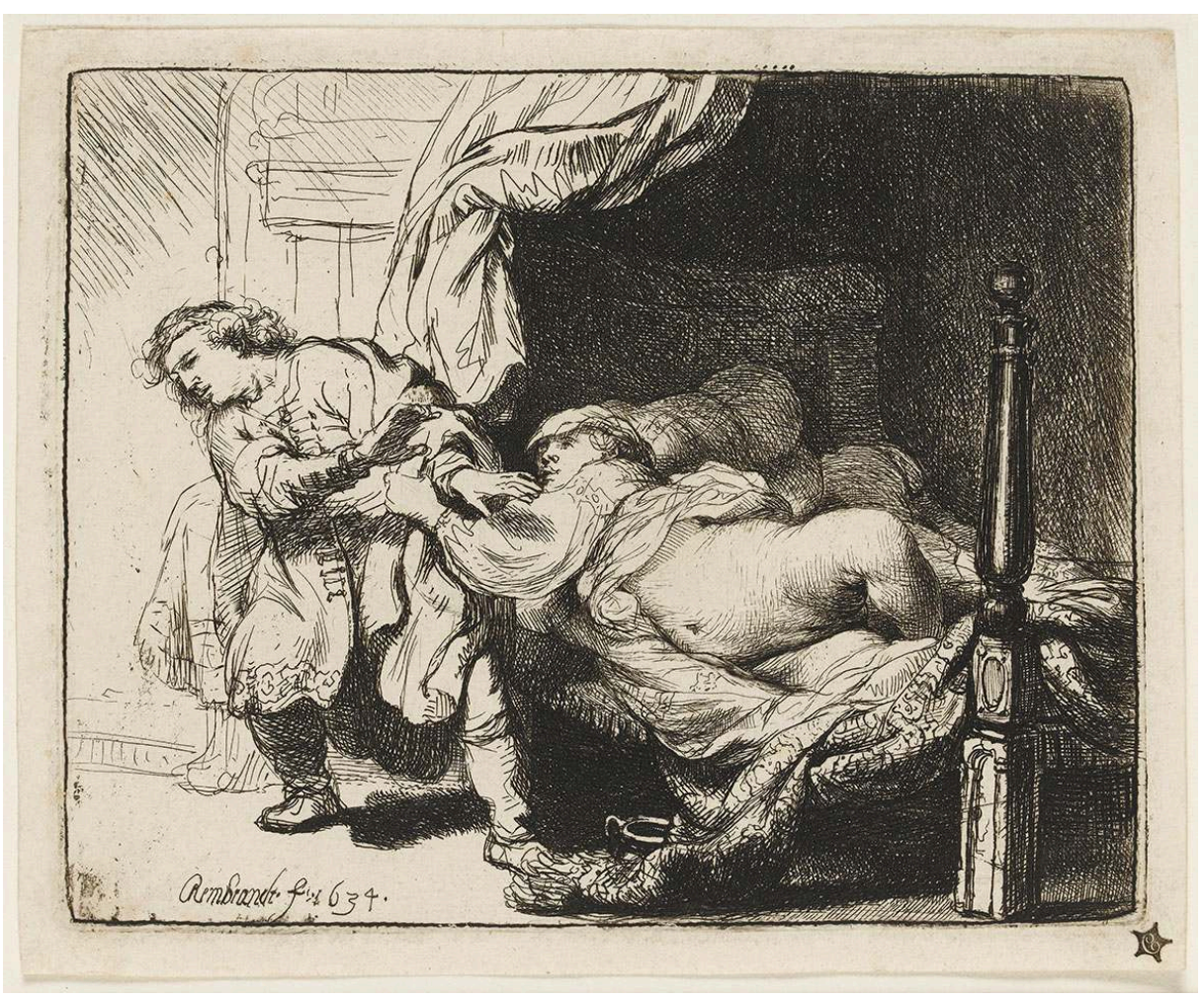

Rembrandt, Joseph et la femme de Putiphar, gravure, 1634, 2225LR. Paris, musée du Louvre, collection Rothschild.

Phot. Thierry Le Mage. (c) RMN-Grand Palais (musée du Louvre).

Enfin, il arrive souvent que la description du lit mentionne une structure «à visse », ce qui indique que la structure est démontable. Le dictionnaire de l'Académie cite d'ailleurs dans ses exemples pour le mot « vis » celle d'un châlit. De nombreux bois de lit en pièces sont ainsi inventoriés dans les garde-meubles des hôtels.

\section{Le coucher}

On désigne aujourd'hui sous le terme de «coucher " l'ensemble des pièces nécessaires au sommeil: sommier, matelas, oreillers, etc. Au xvir siècle, on employait le mot "garniture » pour désigner cet ensemble ${ }^{6}$. Avec le bois et la garniture, il forme le troisième et indispensable élément constitutif du grand lit. À la différence des deux autres, il ne répond qu'à des nécessités de confort et ne participe pas à la valeur d'apparat du meuble. Toutefois, la peine que prennent les notaires à le détailler met bien en évidence son importance dans la valeur du meuble.

Ces descriptions font apparaître une grande variété dans la manière de composer le coucher. Variété qui doit autant être le reflet des moyens du propriétaire que des exigences de confort de l'usager.

Le coucher se présente comme une superposition au nombre varié de strates des pièces qui assurent le confort de la couche. De bas en haut, ces couches apparaissent du plus raide au plus moelleux. 
14 En fonction du type de bois de lit, la couche inférieure varie. Si le bois n'est composé que d'un cadre, le vide central sera comblé par une "enfonçure » fixée au cadre. Il s'agit de "petits ais de 4 ou 5 pouces de large qui sont attachés à quelque distance les uns des autres avec de la sangle, et qu'on étend sur le bois de lit $»^{7}$. Mais ces planches peuvent aussi être solidaires du cadre et appartenir au bois de lit; dans ce cas, il n'y a pas d'enfonçures et l'on pose directement dessus les éléments inférieurs du coucher, la paillasse.

15 Comme l'indique le dictionnaire de Furetière (1690), la paillasse est « la plus basse garniture d'un lit». Il s'agit d'un élément assez grossier, une housse de toile ou de canevas bourrée de paille qui joue principalement un rôle d'isolant thermique.

Dans certains cas, la paillasse peut être remplacée par un « sommier » qui est similaire, mais rempli de crin et non de paille. Cette pièce de tapissier est toutefois plus élaborée puisqu'elle peut être piquée comme un matelas. Mais la fonction est identique à celle de la paillasse, qu'elle remplace avantageusement : selon Richelet, « pour être bien couché il faut avoir un bon matelas, un bon lit de plume, \& un bon sommier de crin au lieu de paillasse».

17 Paillasse ou sommier ne peuvent en aucun cas constituer l'ensemble du coucher. Il faut y ajouter au moins une couche moelleuse, c'est-à-dire une ou plusieurs strates souples, confortables. On distingue ici les «lits» et les «matelas» en fonction des matériaux employés.

Des deux, le matelas est le plus fruste mais aussi le plus fréquent. Au XVII ${ }^{\mathrm{e}}$ siècle, le mot désigne une pièce de tapissier composée de deux grands morceaux d'étoffe qui contiennent entre eux « vingt-cinq ou trente livres de bourre lanice, de laveton ou de laine» (Richelet 1680), cousus le long des bordures et éventuellement piqués. La garniture du matelas peut varier selon la qualité du lit souhaitée - la laine étant, selon Furetière, la plus onéreuse - mais ce qui importe, c'est la densité du matériau contenu entre les deux pièces d'étoffe, le matelas devant assurer autant une fonction d'isolant que de confort. À ce titre, les inventaires sont un peu plus précis que les dictionnaires. On voit donc apparaître l'emploi majoritaire de futaine (étoffe de fil et de coton) pour les enveloppes (parfois de futaine et de toile pour les plus modestes?). Le remplissage est, dans la majorité des cas, de bourre (le terme «bourrelanice " ne se trouve que dans les inventaires des années 1640); le laveton est très rare dans les hôtels. Bourre et laveton sont tous les deux des matériaux grossiers, de second ordre, issus du traitement d'une autre matière, respectivement reliquat de poil provenant du travail des tanneurs, et déchet de laine recueilli après cardage des draps ; Furetière précise que c'est l'étoffe « dont on fait les mauvais matelas ».

19 Comme les matelas, les « lits » sont des « coussins » ou des " couettes », c'est-à-dire des grandes pièces d'étoffe avec un remplissage souple mais réalisés dans des matériaux de meilleure qualité. Le « lit» est constitué de plumes contenues dans du coutil - toile de chanvre ou de lin " extrêmement forte et serrée » (Furetière), spécifiquement utilisée pour les lits, traversins et oreillers. C'est une sorte de matelas plus raffiné, qui prend place au sommet du coucher pour le rendre plus confortable mais n'est pas indispensable, à la différence du matelas.

Enfin, avec le coucher sont systématiquement inventoriés une couverture de laine et un traversin (les draps sont quant à eux toujours prisés à part, avec le linge et les vêtements). On notera que les oreillers sont exceptionnels ${ }^{8}$ et que les traversins sont, 
comme les lits, des pièces de coutil bourrées de plumes. Comme on l'entend aujourd'hui, il s'agit bien d'une « espèce d'oreiller rond qui occupe toute la largeur du lit " (Furetière, 1690), mais les dictionnaires de la fin du siècle le donnent pour synonyme de « chevet de lit ".

Un coucher est donc constitué d'au moins deux éléments principaux, une paillasse et un matelas, l'un assurant un minimum d'isolant thermique et l'autre un minimum de confort. Mais cette association peut être enrichie de diverses manières, pour de multiples raisons. Selon le type de bois, une enfonçure peut apparaître comme strate inférieure ; elle assure un confort particulier, différent d'un fond de planches. Paillasse ou sommier sont quasiment systématiques; ils supportent le matelas. Celui-ci est indispensable; on en trouve jusqu'à trois superposés. Un lit de plumes peut, enfin, former la dernière strate.

Outre l'importance pour le confort du dormeur, la composition du coucher est essentielle pour l'aspect général du lit. En effet, le nombre et le type de strates génèrent une hauteur plus ou moins importante de la couche et par conséquent, des proportions variées de la partie basse du meuble et de sa garniture textile. Dès lors, il devient impossible d'établir une hauteur type.

\section{Les garnitures}

Après l'énumération du coucher, la description des lits dans les inventaires après décès s'attarde à préciser la manière dont il est garni, c'est-à-dire comment tous les éléments précédemment évoqués - bois et coucher - sont dissimulés par une enveloppe textile. Alors qu'elles disparaissent à l'époque contemporaine, ces étoffes sont les plus forts marqueurs des couches anciennes; elles relèvent à la fois du confort et de l'apparat deux notions alors également importantes.

La garniture permet de clore entièrement l'espace de la couche et de créer ainsi autour du dormeur un cocon propice au sommeil, l'isolant de la lumière et l'assurant d'une chaleur constante. Il s'agit de pallier les éventuelles faiblesses de l'architecture de la chambre. Incidemment, elle comporte d'autres avantages, et contribue à créer, pour filer la métaphore proposée par Michèle Perrot à propos de la chambre, " une boîte dans la boîte ", soit un espace " à soi » au sein de la chambre qui est elle-même - dans bien des cas déjà - un espace intime ${ }^{10}$.

Toutefois, dans le corpus particulier qui est le nôtre - les lits des grandes maisons parisiennes -, la garniture revêt dans de nombreux cas une seconde fonction, celle de participer au luxe de l'ameublement de la chambre. Le lit étant le meuble le plus visible dans la pièce et traditionnellement chargé d'une force symbolique importante, sa garniture contribue à faire de la chambre la pièce la plus précieuse de l'appartement et ainsi, à hiérarchiser les espaces de la maison. Sa qualité varie précisément selon le rang de l'occupant de l'appartement : on distingue donc facilement le logement du maître de maison, celui des hommes et des femmes, des parents et des enfants...

Ainsi la garniture de la couche peut-elle relever des mêmes étoffes que les tentures, traditionnellement les tissages les plus précieux de la maison. Toutefois, si elle partage avec la tapisserie ou les soieries sa nature, la garniture de lit suit toujours des usages de composition très codifiés que les inventaires décrivent avec précision. 
Il ressort de l'analyse des descriptions que dans la première moitié du Xvil ${ }^{e}$ siècle à Paris, on rencontre simultanément deux types principaux de garniture de lit. On distinguera ainsi les lits « en housse » des lits munis de rideaux. On notera qu'à leurs côtés, un troisième type directement issu de la Renaissance subsiste, le lit à pavillon, parfois dit «à la romaine ", dont l'importance est grande pour les déclinaisons qui en ont été faites dans la seconde partie du XviI ${ }^{\mathrm{e}}$ siècle.

Les expressions « en housse » ou "à pentes » désignent toutes deux la façon de garnir la couche de manière traditionnelle, par un système simple de grands pans d'étoffe fixés autour du ciel et retombant d'un seul tenant sur chacun des côtés de la couche (fig. 2). Les quatre pans (également dits "pentes» ou "grandes pentes ») entourent ainsi la couche, d'où l'appellation également fréquente de " tour de lit » pour désigner ce type de garniture (dont le principe évoque, à une autre échelle, celui des tapis de table par exemple). L'expression devient ensuite générique pour désigner la garniture des lits.

Figure 2

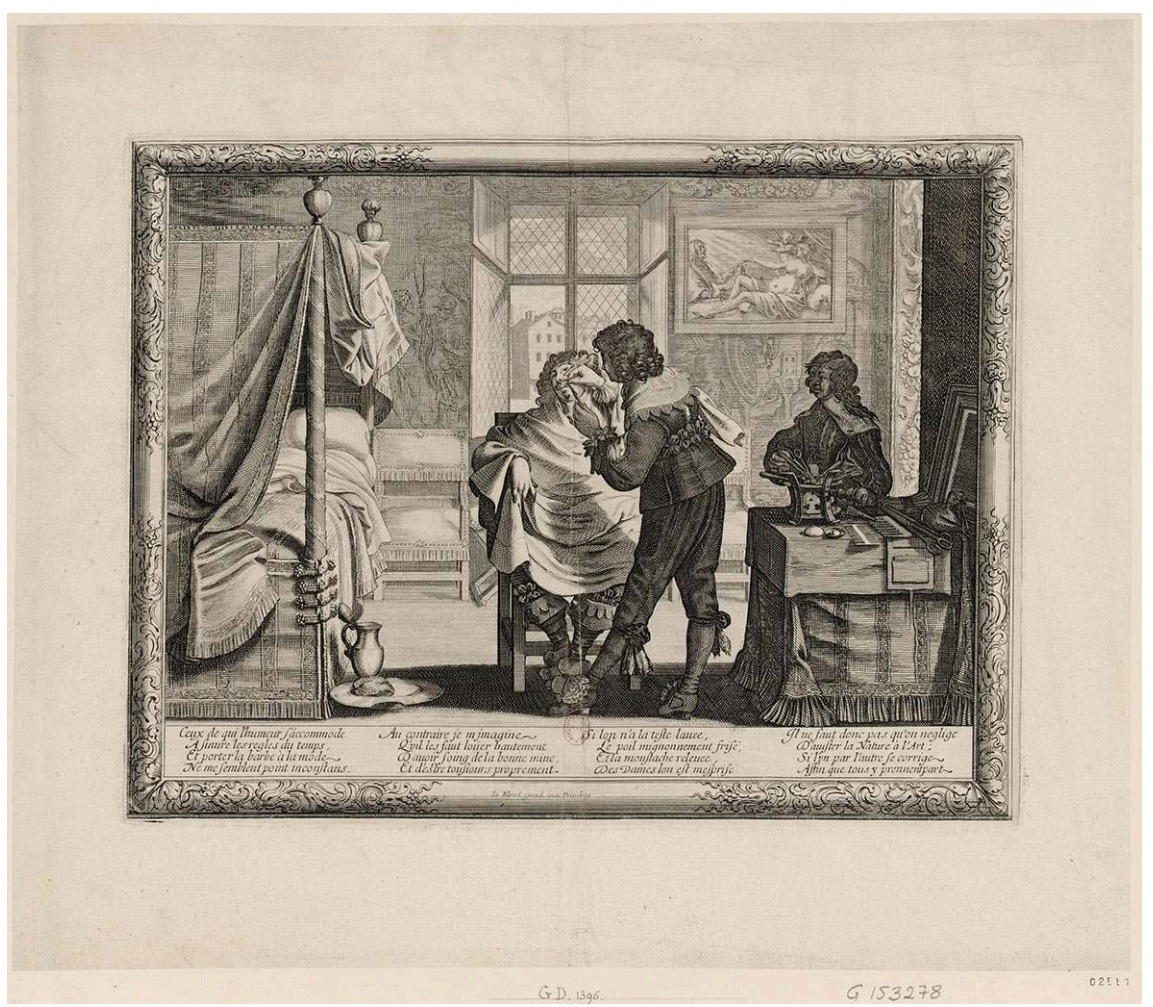

Abraham Bosse, Le Barbier, vers 1632-1633 (BnF : RESERVE FOL-QB-201 (29)). Recueil. Collection Michel Hennin. Estampes relatives à l'Histoire de France. Tome 29, Pièces 2507-2592. Paris, Bibliothèque nationale de France (BnF).

(c) BnF, Dist. RMN-Grand Palais.

Ce système impose la présence de "pommes » au-dessus du ciel. La gravure du Barbier d'Abraham Bosse ${ }^{11}$ montre bien que pour accéder ou sortir du lit, le seul moyen est de rejeter le pan sur le dessus du ciel et de réussir par un geste précis à le retenir par l'une des quatre pommes qui surplombent les piliers. Ce sont des pièces en bois garnies de la même étoffe que le reste de la garniture et qui affectent facilement la forme de vases; 
jouant un rôle fonctionnel, elles ne sont alors pas travaillées de manière spectaculaire ni disproportionnées avec des plumes ou d'autres ornements fragiles.

Ce type est commun et présent dans tous les inventaires de la période étudiée, il est simple et ancien et n'appelle pas une description particulièrement détaillée de la part des notaires. Par exemple, dans la chambre à coucher du duc de Sully, rue SaintAntoine, en 1634: «Un lit en housse de velours cramoisi chamarré de broderie d'or garni de campane, doublé de satin avec la couverture de satin piquée (...) le bois de lit garni d'une paillasse, matelas, traversin et couverture avec les quatre pommes de lit garnies de quatre plumes ${ }^{12}$. »

31 C'est le type de lit qu'Abraham Bosse représente souvent dans ses vues d'intérieurs de la haute bourgeoisie parisiennes des années 1630-1640. Il a l'avantage d'offrir aux tapissiers l'occasion de déployer des grandes pièces et d'orner ainsi la chambre d'un «cube » d'étoffe particulièrement visible - effet spectaculaire qu'Antoine Le Pautre exploite encore dans ses gravures d'alcôves des années 1660 .

Outre la housse, la garniture se compose des pièces d'étoffe assorties qui dissimulent le coucher et le châlit. Ce dernier est entouré par trois pièces qui constituent le "soubassement» et le millefeuille des matelas par la "courtepointe» (parfois aussi simplement désignée "couverture ») dont les pans se superposent au soubassement. Enfin, comme le système des pentes que l'on relève dégage fréquemment les piliers, comme les gravures de Bosse le montrent clairement, ceux-ci - qui ne sont plus guère sculptés à Paris au cours de notre période - sont revêtus de "fourreaux ", toujours des mêmes nature et couleurs que les autres pièces de la garniture. Dans cet ensemble, seule la courtepointe peut être d'une nature différente, éventuellement assortie à la doublure intérieure de la housse. Mais le soubassement et les fourreaux, en tant que pièces extérieures, sont de même étoffe et couleurs que la garniture principale.

L'inconvénient majeur du lit en housse réside naturellement dans son manque de commodité. C'est ce qu'indique en négatif la définition de Furetière (1690): «On appelle (...) le lit en housse celui qui a des pentes qui vont jusqu'en bas ou qui se suspendent sur des bâtons, qui n'a point de rideaux qui se tirent sur des tringles ${ }^{13}$. "

Le second type de lit, que l'on dira "à rideaux » ${ }^{14}$ (fig. 3), apporte une solution à cette incommodité. D'après les inventaires, il coexiste avec le précédent et se rencontre tout au long de la première moitié du siècle - l'évolution d'une typologie à l'autre remonte donc à la fin de la Renaissance. 
Figure 3

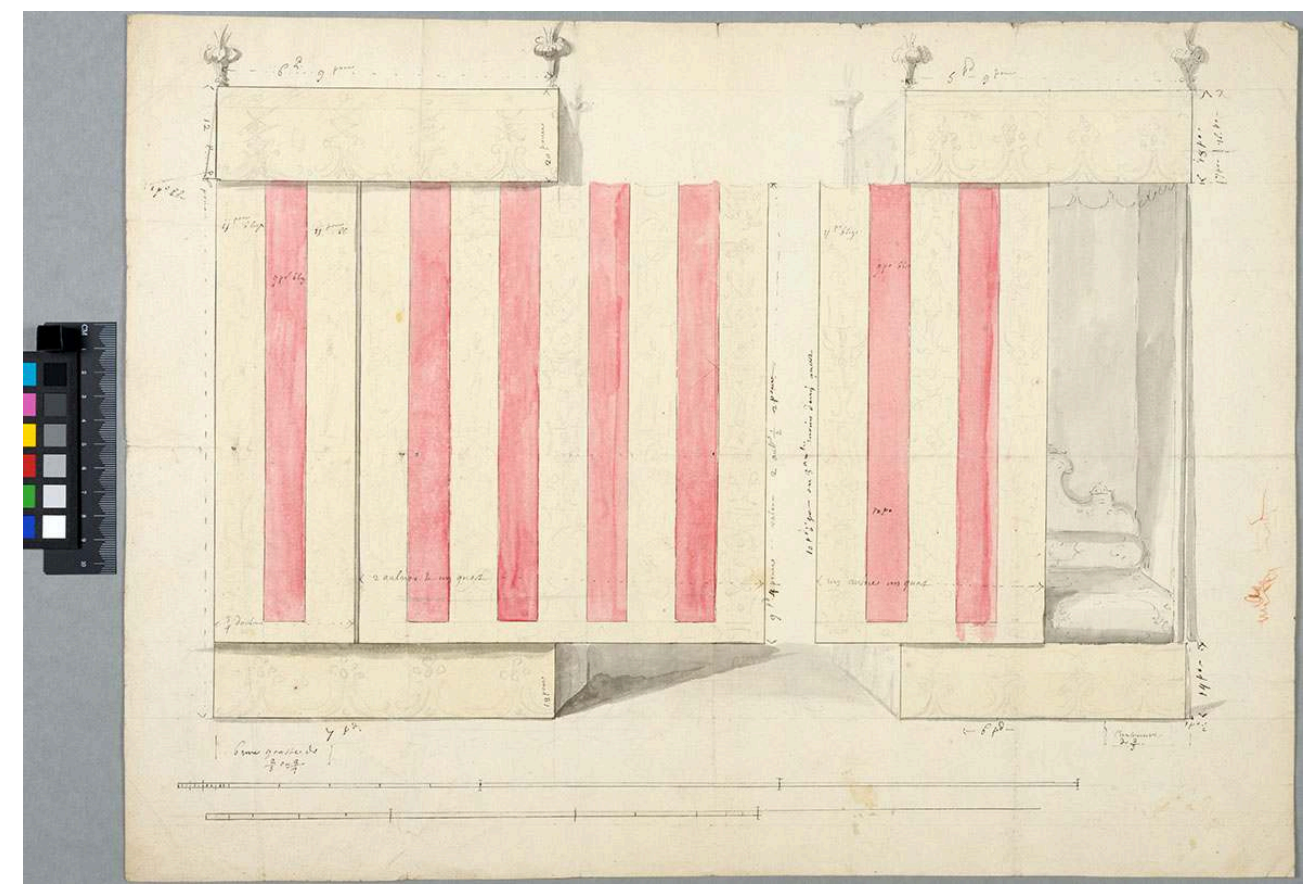

Lit Régence, rideaux fermés et à demi ouverts, dessin anonyme, début du XVIIle siècle. Stockholm, musée national : NMH CC 3025. http://collection.nationalmuseum.se/eMP/eMuseumPlus? service $=$ Externallnterface \&module=collection\&objectld=142577\&viewType=detailView .

(c) Phot. Cecilia Heisser/Nationalmuseum.

On pourra considérer comme représentative cette prisée de la couche trouvée dans la grande chambre de l'hôtel de Miramion ${ }^{15}$ en 1660 :

Une couche à hauts piliers de bois de noyer, fermant à vis, garnie de son enfonçure, une paillasse, un matelas de futaine des deux côtés rempli de bourrelanisse, un lit et traversin de coutil remplis de plumes, une courtepointe et un dossier de satin piqué rouge cramoisi, un fond de petit satin à fleurs piqué [?] de taffetas jaune garni de ouate, trois pentes de ciel, trois rideaux, deux bonne-grâces, deux cantonnières, le tout de velours cramoisi rouge à ramage garni de crépine, frange et molet d'or, quatre pommes dudit lit couvertes de pareille étoffe, passementées et garnies de houppes d'or $(. . .)^{16}$.

Bien que datant sans doute de la seconde moitié du siècle, le dessin conservé à Stockholm sous la cote CC 3025 (voir fig. 3) permet de mieux comprendre les différentes parties de ce type de garniture ainsi que le schéma proposé en annexe.

Voir en annexe: Lexique européen des termes employés pour désigner les différentes parties de la garniture d'un lit à colonnes (" à la française »), XVI ${ }^{\mathrm{e}}$ $\mathrm{XIX}^{\mathrm{e}}$ siècles

La mise en place de rideaux pour faciliter l'accès à la couche a plusieurs incidences sur la composition de la garniture. Comme la définition de Furetière le rappelle, le principe du rideau entraîne l'apparition de tringles. Ces pièces de métal ne pouvant en aucun cas être apparentes, elles sont dissimulées par des pentes fixées autour du ciel, à l'intérieur et à l'extérieur. Ces tringles sont au nombre de trois, puisque les rideaux ne se déploient que sur les grands côtés et au pied du lit. Logiquement, on compte autant de pentes extérieures, alors qu'il peut y avoir quatre pentes intérieures, l'une se superposant au haut du dossier. 

trois côtés, une distinction apparaît pour désigner le grand pan d'étoffe qui subsiste à la tête de la couche, fixe comme auparavant, que l'on désigne comme le $"$ dossier $»^{17}$. Cette pièce peut encore être solidaire de celle, horizontale, qui garnit l'intérieur du ciel, que l'on appelle « fond $»^{18}$.

40 les dissimuler lorsqu'ils sont repliés : les «bonnes-grâces » et les « cantonnières ». Ces deux éléments sont composés de la même manière de pans d'étoffe rigides d'un lé de large environ, placés aux angles du lit. À la tête, les bonnes-grâces sont fixées de chaque côté, alors que les cantonnières sont deux pièces de même largeur, assemblées en angle autour des piliers du pied du lit (fig. 4).

Figure 4

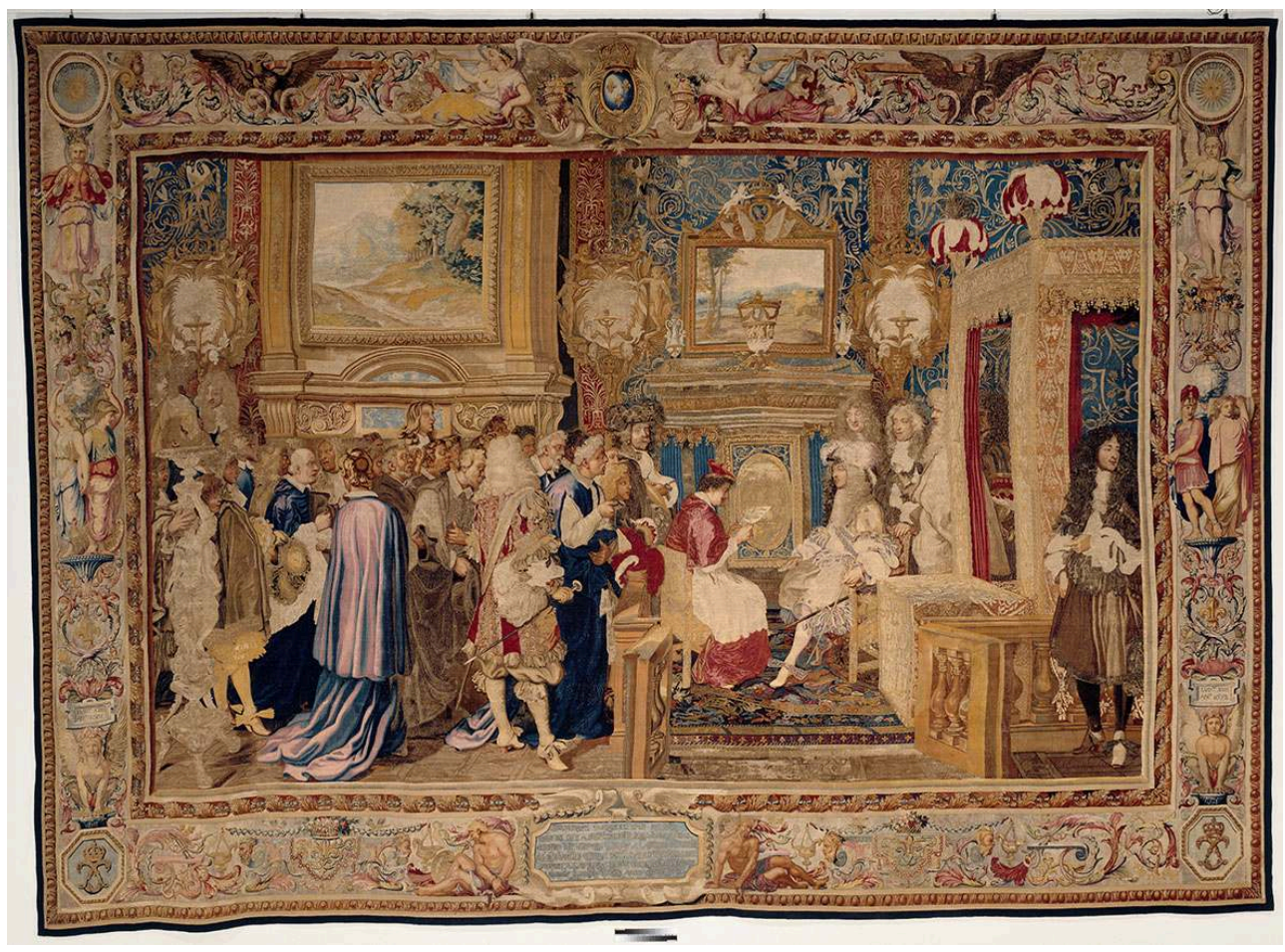

Tenture de l'histoire du Roy, 7e pièce : I'Audience du Légat (29 juillet 1964) ; le cardinal Chigi, vient présenter des excuses au roi Louis XIV dans sa chambre à Fontainebleau. Atelier de Jean Lefebvre, 1667-1672, fil d'or, haute lisse. Paris, musée du Louvre (GMTT95-1).

Phot. Daniel Arnaudet. (c) RMN-Grand Palais (musée du Louvre).

41 Naturellement, il existe de nombreuses variations sur ce schéma de principe. Notamment, selon le type de rideaux employé - en une ou en deux parties -, il y aura ou pas des cantonnières ${ }^{19}$. Il est en outre possible de trouver des cantonnières aux quatre coins de la couche ${ }^{20}$. Dans tous les cas, ces pièces sont faites de la même étoffe que les rideaux et sont garnies de même passementerie. On peut trouver, comme pour le lit en housse, un traitement différencié entre l'intérieur et l'extérieur de la garniture, où les doublures des rideaux et cantonnières sont assorties à la courtepointe, au fond et au dossier. 
Enfin, on notera la persistance des pommes au-dessus du ciel. Désormais inutiles, elles peuvent prendre des proportions plus grandes, des formes plus spectaculaires et faire appel à des matériaux plus délicats - panaches d'aigrettes et de plumes, notamment.

3 Dans le lit à rideaux, la manière de garnir le coucher est identique à celle du lit en housse.

\section{Garnitures exceptionnelles}

4 Aux côtés de ces deux grandes manières de garnir les lits, d'autres typologies apparaissent de manière plus exceptionnelle, comme le lit « en impériale » ou celui « à la romaine ».

Très exceptionnelle, la mention de «lit en impériale» n'apparaît que dans les inventaires dressés à l'hôtel Zamet ${ }^{21}$ en 1614 et 1623 . C'est-à-dire dix mentions sur les 385 couches de notre corpus, soit un groupe très peu représentatif des usages de l'époque.

4 La formule se comprend au regard des textes et de l'iconographie. C'est sans doute Félibien qui donne la définition la plus parlante en 1676 (reprise d'ailleurs par Richelet): "espèce de dôme, ou de couverture dont le dais est en pointe \& qui en s'élargissant par en bas représente la figure de deux $\mathrm{S}$ qui se joignent en haut et s'éloignent en bas (...) ${ }^{22}$.» Pour illustrer son propos, l'auteur cite les carrosses et les toitures de Chenonceau. Ce terme d'architecture a donc glissé jusqu'aux lits pour désigner une forme de ciel complexe, plus architecturée que le simple châssis rectangulaire. L'ampleur ainsi donnée aux lits en a fait, semble-t-il, le type des couches royales; ainsi le lit mis en place dans la nouvelle chambre de Louis XIV à Fontainebleau en 1714 est-il encore "à impérial et à colonnes (...). L'impérial en deux pièces, à l'une desquelles sont attachées les pentes de dedans festonnées ${ }^{23}$ ».

4 Mais la mode est ancienne et le lit de la chambre privée de Sébastien Zamet en 1614 est une couche de bois de noyer façon d'impériale garnie de trois matelas de futaine, un traversin de coutil garni de plume, trois couvertures de Catalogne, dont deux rouges et une blanche, avec son ciel en impériale de drap d'Angleterre bleu chamarré de bandes de broderie et boutons d'or et soie, doublé de taffetas jaune et garni aussi de crépines et franges d'or et soie (210 livres) ${ }^{24}$.

"À l'impériale ", «en façon d'impériale » ou «en impériale ", la formule semble désigner avant tout une silhouette et non pas une structure - le profil chantourné pouvant être atteint aussi bien par la disposition des étoffes en festons ${ }^{25}$ que par l'emploi d'un châssis de menuiserie en forme ${ }^{26}$ (fig. 5). Dans tous les cas, il s'agit d'une variation luxueuse du ciel des couches à quatre piliers. 


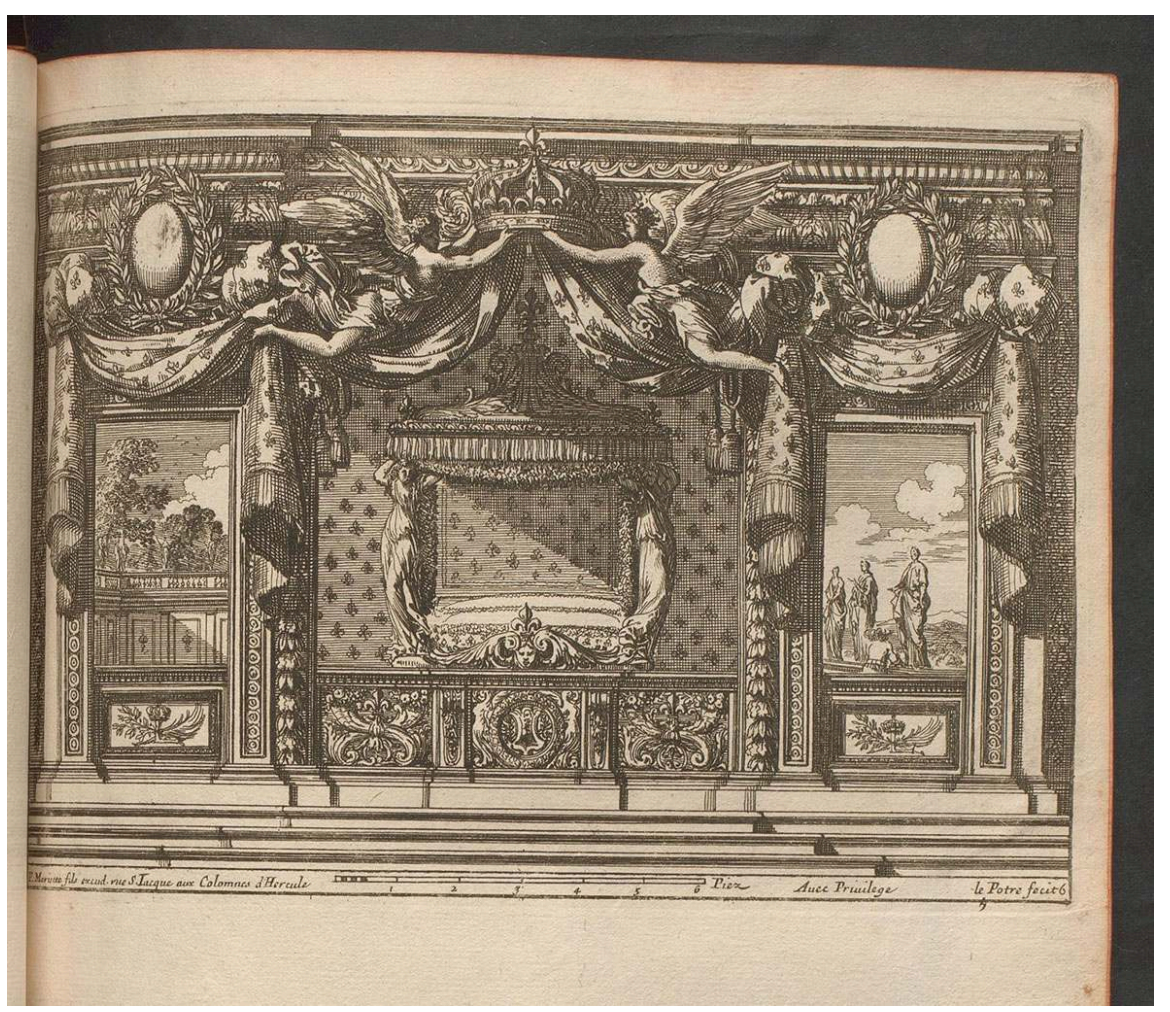

Jean Lepautre, Euvres d'architecture. À Paris : Chez Charles Antoine Jombert, libraire..., 1751, t. 2, n 163. ETH-Bibliothek Zürich, Rar 1331, http://doi.org/10.3931/e-rara-13487 / Public Domain Mark. inventaires pendant toute la période considérée, ils se trouvent toutefois majoritairement, à nouveau, à l'hôtel Zamet. C'est en effet une typologie à la fois archaïque et très fortement marquée par les usages italiens - deux caractères qui dominent la maison de Sébastien Zamet, célèbre banquier piémontais proche de Henri IV. C'est d'ailleurs dans son inventaire que l'on trouve la description très utile d'un "grand pavillon de taffetas bleu fait à la romaine garni de son chapiteau » qui associe le système du pavillon avec l'appellation « à la romaine ».

Ce type représente 35 des couches de notre corpus (soit $9 \%$ ). C'est un type qui s'efface au cours du siècle, au point que le dictionnaire de Richelet, en 1680, précise que «les pavillons ne sont guère en usage que pour les lits des valets ». L'examen des prisées confirme cette tendance : très luxueux dans les inventaires de l'hôtel Zamet de 1614 et 1623 , ils sont ensuite relégués aux pièces de service et ne représentent qu'une faible valeur.

51 Le principe du pavillon est d'être une structure textile totalement indépendante du châssis de la couche et fixée au plafond au-dessus d'elle. Le bois de lit est alors muni de «bas piliers ». Le pavillon se décompose en deux parties : le " chapiteau » et les grandes pentes qui y sont suspendues. À ce titre, il s'apparente aux dais, à la différence que le chapiteau adopte fréquemment une forme circulaire et pyramidale, ce qui lui donne «la figure d'une tente " (Richelet, 1680) comme on le voit dans la riche iconographie des lits de la Renaissance. Les "pentes » sont des lés d'étoffe fixes, descendant jusqu'au sol, qui peuvent envelopper entièrement la couche. Les rares mentions de dimensions font état de hauteurs oscillant entre 2,5 et 3,3 aunes (soit entre $2,95 \mathrm{~m}$ et 
3,90 environ.... $)^{27}$. Leur fixation au chapiteau peut être dissimulée par des petites pentes. Le dictionnaire de l'Académie précise que le pavillon est suspendu " vers le chevet » et non pas au milieu de la couche, entrainnant une sorte de composition décalée qui annonce certains lits $\mathrm{du}$ XVIII ${ }^{\mathrm{e}}$ siècle.

Dans ce système, la cohérence entre les étoffes du pavillon et de la couche semble être moins rigoureuse que dans les cas précédents :

Un grand pavillon de damas de la Chine incarnat contenant 26 pointes sur 3 aunes et $1 / 3$ de haut, une bordure en broderie d'or, garni de dauphins alentour, avec 1 grand chapiteau de velours vert en broderie d'oiseaux et de fleurs profilé d'or, une couverture de parade de velours vert aussi en broderie d'or et figure d'oiseaux et fleurs, deux grands carreaux et quatre petits de même velours et même broderie, un soubassement faisant deux côtés de lit de pareil velours et semblable broderie (750 livres ; garde-meuble de l'hôtel Zamet, 1614)28.

Une mention isolée trouvée dans les inventaires étudiés fait apparaître un usage exceptionnel du pavillon associé à un lit à quatre colonnes :

Une autre garniture de lit contenant le fond, le dossier, les doubles pentes, la couverture de parade, les quatre fourreaux de pilier, trois grands rideaux, le tout de damas violet, les trois pentes de dehors, les trois pentes du soubassement, les quatre cantonnières, le tapis de table, le tout de velours violet, le grand pavillon de damas violet de 24 pointes de 2 aunes $2 / 3$ de haut avec son chapiteau de même tissu, les pentes tant du lit que du pavillon chamarrées de passement d'or et soie violette, garni de franges, crépine, mollet et boutons d'or et le dessous de soie violette » (3 000 livres ; garde-meuble de l'hôtel Zamet, 1614 $)^{29}$.

Selon toute vraisemblance, il s'agit de placer un lit à rideaux sous un pavillon fixé au plafond. Il s'agit apparemment de la reproduction chez un particulier d'un usage qui évoque celui connu dans la sphère royale, où la couche princière est placée sous un dais $^{30}$. La splendeur des étoffes et la présence d'un appartement « du roi » dans l'hôtel de la rue de la Cerisaie peut laisser penser à un lit destiné à Henri IV.

En «impériale » ou "à la romaine ", certaines couches tentent de s'émanciper d'un schéma solidement établi. En apportant à la partie haute une audace formelle facilement spectaculaire et en se libérant de la contrainte des quatre piliers, ces deux types forment séparément les ancêtres du « lit d'ange » qui triomphera dans la seconde partie du siècle (fig. 6). 
Figure 6

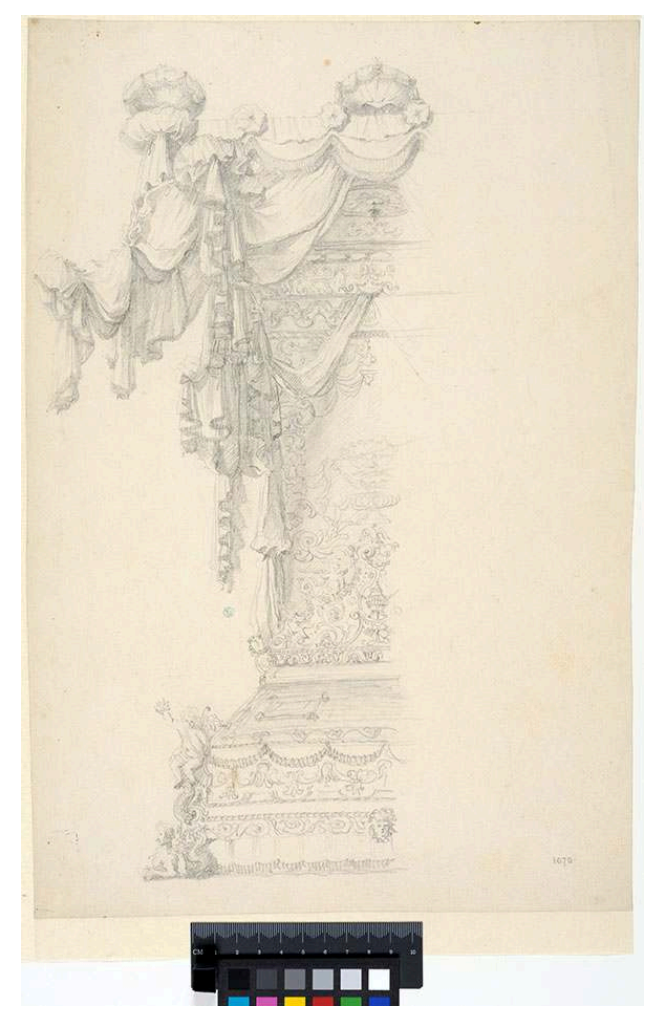

Nicodemus Tessin le jeune (1654-1728), Lit du type utilisé au Trianon de porcelaine. Demi-élévation, dessin. Stockholm, Nationalmuseum : THC 1070. http://collection.nationalmuseum.se/eMP/ eMuseumPlus?

service=Externallnterface \&module=collection\&objectld=146826\&viewType=detailView .

(c) Phot. Cecilia Heisser/Nationalmuseum.

Ce dernier type n'apparaît qu'une fois dans nos inventaires parisiens d'avant 1660 : «Un autre lit d'ange de taffetas de la Chine contenant trois rideaux, quatre cantonnières, trois soubassements, fond, dossier, la courtepointe, le tapis de table, les pommes de lit et sept cordons de soie, le tout garni de petite frange et mollet d'argent » (250 livres ; garde-meuble de l'hôtel Zamet, 1623) ${ }^{31}$.

57 Ce dernier type apparaît comme une variante du lit à pavillon, mais éminemment plus décorative. Pour les dictionnaires de la fin du siècle, leur caractère principal est de s'être affranchi des piliers, mais ce qui les définit également, c'est la richesse de la garniture et «les rideaux qui sont liez avec des rubans faisant d'agréables nœuds» pouvant former une superstructure spectaculaire.

58 Sous le règne de Louis XIV, ce seront les lits d'apparat par excellence et la manifestation la plus "baroque» des métamorphoses des couches au XVII siècle. Aujourd'hui malheureusement entièrement perdus, les lits d'ange sont ainsi le jalon oublié de l'invention du lit à la duchesse qui s'impose à la fin des années $1690^{32}$. 


\section{NOTES}

1. - Le Dictionnaire de l'Académie en 1694 rappelle en effet que le lit est un « meuble dont on se sert pour y dormir ".

2. - Le Dictionnaire de l'Académie françoise. T. 1. Paris : chez Jean-Baptiste Coignard, 1694.

3. - Nous utilisons pour cette étude une sélection parmi le corpus que nous avons constitué d'inventaires après décès réalisés dans des hôtels particuliers parisiens au XVII siècle dans le cadre de notre thèse de doctorat (voir COURTIN, Nicolas. L'Art d'habiter. Dijon : Faton, 2011 ; les actes sont consultables sur le site du Centre André Chastel: http://www.centrechastel.parissorbonne.fr/page/corpus-des-hotels-parisiens-du-xviie-siecle-inventaires-apres-deces-de-24-

hotels [consulté le 02/07/2019]). Nous avons retenu les actes dressés entre 1610 et 1660, dans lesquels sont mentionnés 385 lits.

4. - 9 mentions sur les 385 lits inventoriés dans notre corpus.

5. - Voir plus bas, les lits « à la romaine».

6. - À la différence d'aujourd'hui où ce mot désigne plus particulièrement l'ensemble des textiles qui ferment le lit.

7. - RICHELET, Pierre. Dictionnaire françois. Paris : chez Jean Herman Widerhold, 1680.

8. - Une seule mention trouvée dans notre corpus.

9. - Richelet 1680 : «Chevet de lit ». Furetière 1690 : «Chevet d'un lit, espèce d'oreiller rond qui occupe toute la largeur du lit, qu'on fait ordinairement de coutil rempli de plume». Académie 1694 : « Chevet de lit : sorte d'oreiller rond, et aussi long que le lit est large.»

10. - PERROT, Michelle. Histoire de chambres. Paris : Éd. du Seuil, 2009, notamment p. 53-85.

11. - Abraham Bosse. Le Barbier, estampe, vers 1632-1633.

12. - Inventaire de Maximilien de Béthune (AN, MC/III/554, 5 mai 1634).

13. - Nous soulignons.

14. - Plutôt que « à la française » tellement ce type est commun à l'ensemble de l'Europe.

15. - 47, quai de la Tournelle ( $5^{\mathrm{e}}$ arr.).

16. - Inventaire après le décès de Christophe Martin (AN, MC/LXV/51, 13 avril 1660).

17. - On notera que le terme "dossier » ne désigne qu'une pièce textile et non pas un châssis garni.

18. - Voir par exemple, ce lit du garde-meuble de l'hôtel Zamet avec «le dossier façon de pente [et] le fond cousu ensemble » (1623). Le « fond » désigne uniquement la pièce textile qui garnit le « ciel », qui est la structure supérieure du lit.

19. - « Une garniture de lit contenant le fond, le dossier et trois pentes, le tout de broderie d'or et d'argent relevé en brosse à figures d'or uni avec perles [?] représentant l'histoire d'Apollon et des neufs Muses, trois grands rideaux et deux bonne grâces de damas cramoisi chamarré de passement d'or et soie cramoisi sur les coutures, une courtepointe de satin cramoisi piquée et emboutie et profilé d'or, les pentes garnies de houppes d'or et soies cramoisi » (3000 livres ; garde-meuble de l'hôtel Zamet, 1614 (AN, MC/XIX/381, 13 août 1614).

20. - «Une couche de bois de noyer garnie de son enfonçure fermant à vis, trois matelas dont deux de futaine remplie de bourrelanisse et un autre de toile plein de laveton, un traversin de futaine blanche garni de duvet, un lit de velours bleu à double pentes, savoir six pentes, le fond, dossier, deux fourreaux, quatre cantonnières, la couverture de soubassement, le tout de velours bleu et trois rideaux de damas, le tout chamarré sur les demi lés de passement d'or fort large, frange et crépine, garni de boutons et boutonnières, une couverture de taffetas bleu telle quelle à mettre sous la couverture de velours, quatre pommes de velours chamarré de passement d'or avec leurs bouquets (...)»; grand cabinet de l'hôtel de Marle, 1610 (inventaire après le décès d'Élisabeth Dolu : AN, MC/CV/230, 26 août 1610). 
21. - Anciennement rue de la Cerisaie ( $4^{\mathrm{e}}$ arr.).

22. - FÉLIBIEN, André. Des principes de l'architecture, de la sculpture, de la peinture et des autres arts qui en dépendent, avec un dictionnaire des termes propres à chacun de ces arts. Paris : chez Jean-Baptiste Coignard, 1676, p. 624.

23. - Meuble $\mathrm{n}^{\circ} 2118$ de l'inventaire général du mobilier de la Couronne, livré par le Sr Lallié le 14 août 1714 (AN, 01/3408, fol. $154 \mathrm{v}^{\circ}$ et $155 \mathrm{r}^{\circ}$ ).

24. - Inventaire après le décès de Sébastien Zamet (AN, MC/XIX/381, 13 août 1614).

25. - Voir par exemple : Maître des cassoni Campana, Ariane à Naxos, entre 1510 et 1520, peinture sur bois (Avignon, musée du Petit Palais, MI 529).

26. - Voir par exemple certains lits représentés dans les alcôves de Le Pautre.

27. - Inventaire après le décès de Sébastien Zamet (AN, MC/XIX/381, 13 août 1614).

28. - Ibid.

29. - Ibid.

30. - Voir la gravure anonyme représentant la chambre de Marie de Médicis à St James's Palace, Westminster, Londres, en 1639.

31. - Inventaire après le décès de Jean Zamet (AN, MC/XIX/390, 19 janvier 1623).

32. - COURTIN, N. Op. cit., p. 210.

\section{RÉSUMÉS}

La question du vocabulaire est essentielle dans l'étude des lits de l'époque moderne. Par son importance, la complexité de ses compositions et la généralisation à travers les territoires d'un même modèle, la langue française a plusieurs mots pour décrire cet élément de mobilier. En se fondant sur le dépouillement d'actes notariés parisiens de la première moitié du XVII ${ }^{\mathrm{e}}$ siècle, nous mettons en lumière le vocabulaire - officiel en quelque sorte - utilisé dans la capitale pour décrire ce meuble essentiel. L'analyse des mots met ainsi en évidence l'existence de plusieurs typologies de lits et de garnitures dans les intérieurs parisiens du règne de Louis XIII et de la régence d'Anne d'Autriche. La description des bois de lit permet de distinguer les couches à quatre piliers de celles sans ciel, ou d'avoir une idée des différentes tailles de ce meuble. L'examen des différents éléments qui composent le coucher établit qu'il n'y a aucune règle dans la manière de superposer les matelas, lits et paillasse pour composer une couche - qu'elle soit d'apparat ou d'usage quotidien. Enfin, deux façons coexistent pour garnir les lits, avec ou sans rideaux; distinction qui entraîne une composition plus ou moins complexe et une démultiplication des éléments et donc du vocabulaire. Enfin, cette réflexion sur le vocabulaire permet d'attirer l'attention sur les évolutions sémantiques qui ont eu lieu entre le XVII ${ }^{\mathrm{e}}$ siècle et le nôtre et dont il faut être averti pour comprendre et restituer les lits anciens.

The question of vocabulary is an essential one in the study of beds during the early modern period. Because of its importance, the complexity of its composition and its generalisation throughout the country, it was a piece of furniture for which there were several words in the French language. Based on an analysis of notarial deeds in Paris during the first half of the seventeenth century, this article brings to light the 'official' vocabulary, so to speak, used in the French capital to designate this essential item of furniture. This analysis of the relevant vocabulary indicates the existence of several types of beds and bed linen in the Paris interiors of the reign of Louis XIII and the regency of Anne of Austria. The description of the wooden frames 
allows a preliminary distinction to be made between four-poster beds and those without any covering, and gives an idea of the different sizes the bed might have. The examination of different elements of the bed suggests that there were no clear rules for the way bed frames, mattresses and straw were assembled to make an object in which to sleep, whether for stately occasions or daily use. Finally, there were two main ways for the bed to be garnished, with or without curtains. These distinctions all make for a broad variety in the ways of putting the bed together, a multiplication of the number of elements involved, and, consequently, of the elements of vocabulary. This analysis of the vocabulary involved allow us to draw attention to semantic evolutions between the seventeenth century and the present day, evolutions that have to be taken into account in order to understand and recreate historical beds.

\section{INDEX}

Keywords : bed, seventeenth century, vocabulary, furniture, Paris (France), town house, inventory after death, habitat, seventeenth-century textiles

Mots-clés : lit, xviie siècle, vocabulaire, ameublement, Paris (France), hôtel particulier, inventaire après décès, habitat, textile xviie siècle

\section{AUTEUR}

\section{NICOLAS COURTIN}

Archives de Paris nicolas.courtin@paris.fr 